\title{
Healthcare Utilization and End-of-Life Outcomes in Patients Receiving CAR T-Cell Therapy
}

\author{
P. Connor Johnson, MD ${ }^{1, *}$; Caron Jacobson, MD, MMSc ${ }^{2, *}$; Alisha Yi'; Anna Saucier, $\mathrm{MS}^{2}$; Tejaswini M. Dhawale, MD; \\ Ashley Nelson, PhD; ; Mitchell W. Lavoie, BS3; Mathew J. Reynolds, BA33; Carlisle E.W. Topping, BA3; \\ Matthew J. Frigault, MD'; and Areej El-Jawahri, MD
}

\section{ABSTRACT}

Background: CAR T-cell therapy has revolutionized the treatment of patients with hematologic malignancies, but it can result in prolonged hospitalizations and serious toxicities. However, data on the impact of CAR T-cell therapy on healthcare utilization and end-of-life (EOL) outcomes are lacking. Methods: We conducted a retrospective analysis of 236 patients who received CAR T-cell therapy at 2 tertiary care centers from February 2016 through December 2019. We abstracted healthcare utilization and EoL outcomes from the electronic health record, including hospitalizations, receipt of ICU care, hospitalization and receipt of systemic therapy in the last 30 days of life, palliative care, and hospice referrals. Results: Most patients (81.4\%; $\mathrm{n}=192$ ) received axicabtagene ciloleucel. Overall, $28.1 \%$ of patients experienced a hospital readmission and $15.5 \%$ required admission to the ICU within 3 months of CAR T-cell therapy. Among the deceased cohort, $58.3 \%$ (49/84) were hospitalized and 32.5\% (26/80) received systemic therapy in the last 30 days of life. Rates of palliative care and hospice referrals were $47.6 \%$ and $30.9 \%$, respectively. In multivariable logistic regression, receipt of bridging therapy (odds ratio [OR], 3.15; $P=.041$ ), index CAR-T hospitalization length of stay $>14$ days (OR, 4.76; $P=.009)$, hospital admission within 3 months of CAR T-cell infusion (OR, 4.29; $P=.013)$, and indolent lymphoma transformed to diffuse large B-cell lymphoma (OR, 9.83; $P=.012)$ were associated with likelihood of hospitalization in the last 30 days of life. Conclusions: A substantial minority of patients receiving CAR T-cell therapy experienced hospital readmission or ICU utilization in the first 3 months after CAR T-cell therapy, and most deceased recipients of CAR T-cell therapy received intensive EoL care. These findings underscore the need for interventions to optimize healthcare delivery and EoL care for this population.

J Natl Compr Canc Netw 2021;19(8):928-934 doi: $10.6004 /$ jnccn.2020.7678

${ }^{1}$ Department of Medicine, Division of Hematology \& Oncology, Massachusetts General Hospital Cancer Center \& Harvard Medical School; ${ }^{2}$ Department of Medical Oncology, Center for Lymphoma, Dana-Farber Cancer Institute \& Harvard Medical School; and ${ }^{3}$ Department of Psychiatry, Massachusetts General Hospital \& Harvard Medical School, Boston, Massachusetts.

*These authors contributed equally as co-first authors.

\section{Background}

CAR T-cell therapy is a novel treatment that involves altering the patient's autologous $\mathrm{T}$ cells to target a cell surface antigen on the tumor. ${ }^{1,2}$ CAR T-cell therapy has transformed the treatment of relapsed/refractory large B-cell lymphomas. ${ }^{3-5}$ However, patients receiving this treatment are often hospitalized and experience unique toxicities, such as cytokine release syndrome (CRS) and immune effector cell-associated neurotoxicity syndrome (ICANS). These toxicities can result in high rates of ICU admissions and prolonged lengths of stay (LoS) in the hospital. ${ }^{4-10}$

Despite the revolutionary nature of CAR T-cell therapy and its treatment-related toxicity, we lack a basic understanding of healthcare utilization in this population. Given the potential for remission despite their rapidly progressive disease, patients undergoing cellular therapy are at risk for intensive healthcare utilization and face immense prognostic uncertainty. It remains unclear to which population cellular therapy may offer a curative potential, leaving patients unsure about their future health. Unfortunately, approximately $50 \%$ of patients experience disease progression and die within 6 months of CAR T-cell therapy. ${ }^{4,5,11}$ However, data describing healthcare utilization and end-of-life (EoL) outcomes in this population are lacking.

In the present study, we sought to describe the healthcare utilization and EoL outcomes among patients treated with CAR T-cell therapy. We also aimed to examine associations among patient and clinical characteristics and important EoL outcomes. Data depicting patients' healthcare utilization and EoL care could allow clinicians to communicate important information about the ramifications of treatment and provide patients with critical information to plan for the future. Understanding factors associated with intensive EoL care can be instrumental in identifying a high-risk population that may benefit from interventions to optimize their EoL care. 


\section{Methods}

\section{Study Design}

We conducted a retrospective analysis of adult patients treated with CAR T-cell therapy at Dana-Farber Cancer Institute (DFCI) or Massachusetts General Hospital (MGH) between February 2016 and December 2019. We excluded patients who were seen for consultation but did not receive CAR T-cell therapy at either institution. We identified the eligible cohort through the MGH and DFCI CAR-T therapy database, which includes all patients receiving CAR T-cell therapy at our institutions. The Dana-Farber/Harvard Cancer Center Institutional Review Board approved this study.

\section{Clinical Information}

We abstracted information from the electronic health record (EHR) through a comprehensive chart review about patients' demographics; ECOG performance status (determined within 2 weeks of CAR T-cell infusion); diagnosis; dates of relapse and CAR T-cell infusion; therapies received; CAR T-cell product; presence and grade of toxicities, including CRS and ICANS; receipt of tocilizumab and/or corticosteroids; response to treatment; and duration of follow-up. For patients with $\geq 1$ year of follow-up, we determined the rate of survival at 1 year after CAR T-cell therapy infusion.

\section{Healthcare Utilization and EoL Care}

We obtained information regarding LoS, frequency and dates of hospital readmission, and ICU admissions from the EHR. We calculated the rates of ICU admission during CAR T-cell therapy treatment and the rates of ICU admission and hospital readmission within 3 months of CAR T-cell therapy infusion. We reviewed the discharge summaries of hospital readmissions to determine the primary reason for each hospital readmission. We adapted a coding schema previously developed in patients with leukemia to determine reasons for hospital readmissions. ${ }^{12}$ In the schema for our study, these reasons included symptoms, fever without a source, febrile neutropenia, confirmed infection, dehydration/ electrolyte abnormalities, planned hospitalization, hospitalization due to a noncancer medical condition, cancer progression, and hospitalization due to CAR T-cell toxicities (CRS and ICANS). ${ }^{12,13}$ We used symptoms as the reason for hospital admission when the admission was for symptom management; all other causes of admission were excluded or no primary etiology of the admission was defined.

We determined patients' place of death, cause of death, palliative care utilization, hospice utilization, and LoS in hospice using the EHR and the Social Security Death Index. We also determined whether patients were hospitalized (yes vs no), received systemic therapy (yes vs no), or were admitted to the ICU (yes vs no) within 30 days of death. For most patients receiving CAR-T therapy, the healthcare utilization and EoL care occurred within our system. Additionally, the clinical team maintaining the CAR-T database obtains information on healthcare utilization and EoL outcomes at other institutions and these are scanned into the EHR to maintain high data quality.

\section{Statistical Analysis}

We used descriptive statistics to summarize patients' sociodemographic and clinical characteristics, rates of toxicities and response, and rates of survival at 1 year after CAR T-cell therapy infusion. We used descriptive statistics to describe healthcare utilization for all patients in this cohort and to characterize EoL outcomes for patients who died in the cohort.

We used multivariable logistic regression to examine the association between patient demographics and clinical factors and binary outcomes of interest (hospitalization within 30 days of death and hospice referral). We examined factors associated with these outcomes given they are important outcomes in EoL care. ${ }^{14}$ We first conducted univariate analyses to assess the association between patient demographic (eg, marital status) and clinical factors (ECOG performance status, index hospitalization LoS $>14$ days, prior therapies received, prior autologous stem cell transplant, CAR T-cell product, ICU admission within 3 months after CAR T-cell infusion, hospital admission within 3 months after CAR T-cell infusion, CRS, ICANS, receipt of tocilizumab and/or steroids, disease response, and palliative care consultation) with the binary outcomes of interest. Variables with a $P$ value $<.10$ in the univariate analyses were included in the multivariable models. ${ }^{15,16}$ In the multivariate model of hospitalization within 30 days of death, we forced age, sex, receipt of bridging therapy, and disease subtype into the model a priori because these variables have been associated with EoL outcomes. In the multivariate model of hospice referral, we included age, sex, and disease subtype a priori because of their known association with hospice utilization. ${ }^{17-19}$ All reported $P$ values are 2-sided with a value of $<.05$ considered statistically significant. We performed statistical analyses using STATA, version 14.2 (StataCorp LP).

\section{Results}

\section{Study Participants}

Table 1 describes the sociodemographic and clinical characteristics of the study cohort $(n=236)$. Median age was 62.5 years, and most patients were male $(n=145$; $61.4 \%)$, White $(\mathrm{n}=218 ; 92.4 \%)$, and married/had a life 


\section{Table 1. Patient Characteristics}

\begin{tabular}{|lc|}
\hline Characteristic & $\mathbf{n}(\%)$ \\
\hline Total, $\mathrm{n}$ & 236 \\
\hline Age, median (range), y & $62.5(19-82)$ \\
\hline Female sex & $91(38.6)$ \\
\hline White race & $218(92.4)$ \\
\hline Relationship status & $163(69.1)$ \\
\hline Married/Life partner & $40(17.0)$ \\
\hline Single & $13(5.5)$ \\
\hline \begin{tabular}{l} 
Divorced/Legally separated \\
\hline Widowed
\end{tabular} & $13(5.5)$ \\
\hline Unknown & $7(3.0)$ \\
\hline CAR T-cell product & $183(77.5)$ \\
\hline \begin{tabular}{l} 
Axicabtagene ciloleucel \\
\hline Tisagenlecleucel
\end{tabular} & $36(15.3)$ \\
\hline $\begin{array}{l}\text { Axicabtagene ciloleucel combined with } \\
\text { immunotherapy }\end{array}$ & $9(3.8)$ \\
\hline KTE-X19 & $7(3.0)$ \\
\hline Lisocabtagene maraleucel & $1(0.4)$ \\
\hline Lymphoma subtype & $107(45.3)$ \\
\hline DLBCL/Grade 3B follicular lymphoma & $40(17.0)$ \\
\hline $\begin{array}{l}\text { Indolent lymphoma transformed to DLBCLb } \\
\text { rearrangements }\end{array}$ & $40(17.0)$ \\
\hline Follicular lymphoma & $12(5.1)$ \\
\hline Other & \\
\hline
\end{tabular}

(continued)

partner $(n=163 ; 69.1 \%)$. Most patients $(n=194 ; 82.2 \%)$ had an ECOG performance status of 0 or 1 . The most common lymphoma subtype was diffuse large B-cell lymphoma (DLBCL) or grade 3B follicular lymphoma $(\mathrm{n}=107 ; 45.3 \%)$, and the median prior lines of therapy was 3 . Overall, $81.4 \%$ of patients $(n=192)$ received axicabtagene ciloleucel, 39.4\% $(\mathrm{n}=93)$ received bridging therapy, and $27.5 \%(n=65)$ had a prior autologous stem cell transplant.

In terms of toxicities, $77.5 \%$ of patients $(n=183)$ had CRS, with $5.9 \%(\mathrm{n}=14)$ having grade $\geq 3$ CRS. In addition, $53.4 \%$ of patients $(n=126)$ had ICANS, with $22.0 \%(n=52)$ having grade $\geq 3$ ICANS. Approximately one-half of patients $(\mathrm{n}=120 ; 50.9 \%)$ received tocilizumab, and $45.3 \%(\mathrm{n}=107)$ received corticosteroids. The overall response rate was $85.2 \%(n=201)$, the complete response rate was $64.4 \%$ $(\mathrm{n}=152)$, and $63.0 \%(116 / 184)$ of evaluable patients were alive 1 year after CAR T-cell therapy. Overall, $60.6 \%$ of patients experienced a complete or partial response and did not experience disease progression, $24.6 \%$ had an initial response followed by subsequent progression,

\begin{tabular}{|c|c|}
\hline Characteristic & n (\%) \\
\hline \multicolumn{2}{|l|}{ ECOG performance status } \\
\hline $0-1$ & $194(82.2)$ \\
\hline $2-4$ & $38(16.1)$ \\
\hline Unknown & $4(1.7)$ \\
\hline Received bridging therapy & $93(39.4)$ \\
\hline Prior lines of therapy, median (range) & $3(0-10)$ \\
\hline Prior autologous stem cell transplant & $65(27.5)$ \\
\hline $\begin{array}{l}\text { Days from relapse to CAR T-cell therapy, } \\
\text { median (range) }\end{array}$ & $58(11-391)$ \\
\hline \multicolumn{2}{|l|}{ Toxicity and response } \\
\hline Cytokine release syndrome (any grade) & $183(77.5)$ \\
\hline ICANS (any grade) & $126(53.4)$ \\
\hline Received tocilizumab & $120(50.9)$ \\
\hline Received corticosteroids & $107(45.3)$ \\
\hline Overall response & $201(85.2)$ \\
\hline Complete response & $152(64.4)$ \\
\hline Survival & 184 \\
\hline Alive 1 year after CAR T-cell therapy ${ }^{d}$ & $116(63.0)$ \\
\hline
\end{tabular}

Abbreviations: DLBCL, diffuse large B-cell lymphoma; $\mathrm{HGBCL}$, high-grade B-cell lymphoma; ICANS, immune effector cell-associated neurotoxicity syndrome.

a3 patients either had missing data or declined to report for race.

bRichter transformation was classified under other.

${ }^{5} 5$ patients had missing data.

${ }^{\mathrm{d}}$ Including only evaluable patients.

and $14.8 \%$ had stable disease or experienced progression despite receipt of CAR T-cell therapy. Among patients with a complete or partial response and no subsequent relapse, $6.3 \%(9 / 143)$ had grade $\geq 3$ CRS, $25.2 \%$ (36/143) had grade $\geq 3$ ICANS, and $86.9 \%$ (86/99) of evaluable patients were alive 1 year after CAR T-cell therapy.

\section{Healthcare Utilization}

Table 2 describes the healthcare utilization of patients receiving CAR T-cell therapy in our cohort. Median LoS for CAR T-cell therapy hospitalization was 15 days, and $12.7 \%$ of patients (30/236) required an ICU admission during the hospitalization. Overall, $15.5 \%$ of patients $(36 / 232)$ had an ICU admission within 3 months of CAR T-cell infusion, and $28.1 \%(65 / 231)$ had at least one hospital readmission within 3 months of CAR T-cell infusion. The median time from discharge to first hospital readmission was 18 days. Among patients with a complete or partial response and no subsequent relapse, $15.0 \%(21 / 140)$ had an ICU admission within 3 months of CAR T-cell infusion, and $23.7 \%$ (33/139) had at least one hospital readmission within 3 months of CAR T-cell infusion. 


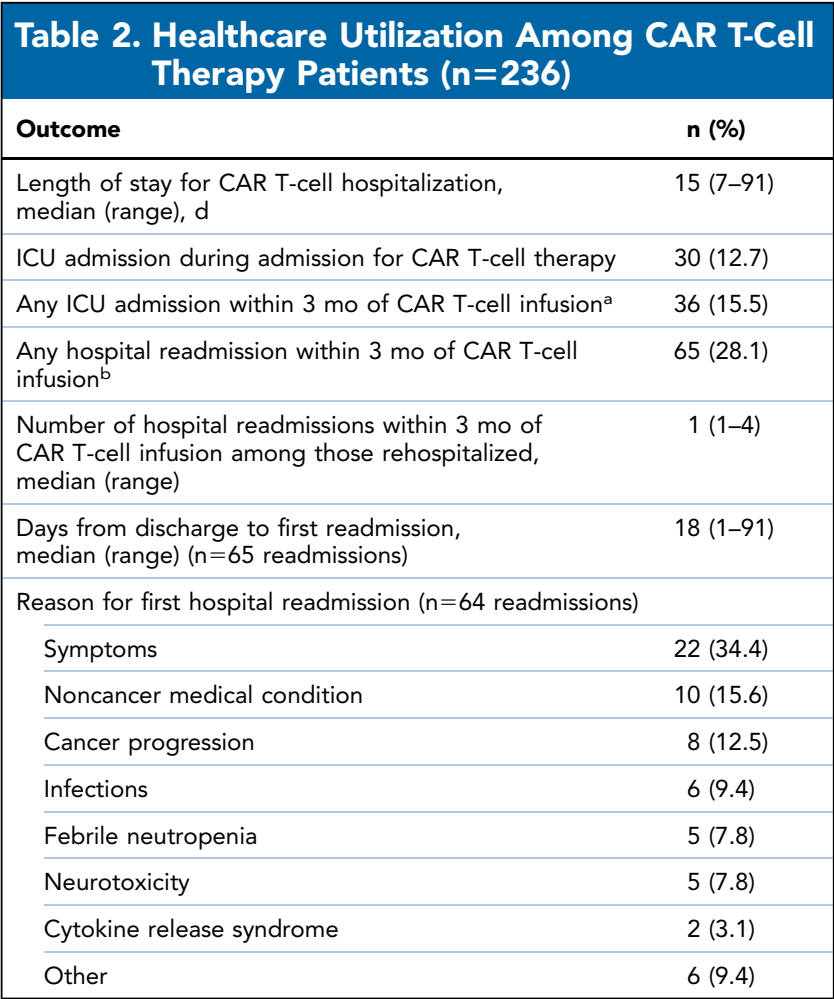

a4 patients had missing data.

b5 patients had missing data.

The most common reason for first hospital readmission was patient symptoms (22/64; 34.4\%; Table 2). Among those admitted for symptoms, the most common were pain $(27.3 \%)$, fatigue/weakness $(18.2 \%)$, and confusion (13.6\%). For those admitted for noncancer medical conditions, among the most common causes were transient ischemic attack (10\%), hematoma (10\%), and incarcerated hernia (10\%).

\section{EoL Outcomes}

Table 3 provides the EoL outcomes among the cohort of deceased patients $(n=84)$. Within 30 days of death, $58.3 \%$ (49/84) were hospitalized, $32.5 \%(26 / 80)$ received systemic therapy, and $11.9 \%(10 / 84)$ were admitted to the ICU. Among the deceased cohort, $47.6 \%$ $(40 / 84)$ had a palliative care consultation, and a minority $(18 / 82 ; 22.0 \%)$ received palliative care $>30$ days before death. Only $30.9 \%$ (25/81) received hospice services, and most $(62 / 80 ; 77.5 \%)$ had a hospice $\operatorname{LoS} \leq 7$ days. Among all deceased patients, $36.9 \%(\mathrm{n}=31)$ died in a hospital, rehabilitation facility, or nursing home; 20.2\% $(n=17)$ died at home; $7.1 \%(n=6)$ died in inpatient hospice; and $35.7 \%(n=30)$ had an unknown place of death. The most common cause of death was cancer progression $(58 / 84 ; 69.1 \%)$. Within 30 days of death among 19 evaluable patients with a complete or partial response and no subsequent relapse, $42.1 \%(n=8)$ were

\begin{tabular}{|c|c|}
\hline End-of-Life Outcome & n (\%) \\
\hline Hospitalization within $30 \mathrm{~d}$ of death & $49(58.3)$ \\
\hline Chemotherapy within $30 \mathrm{~d}$ of death ${ }^{\mathrm{a}}$ & $26(32.5)$ \\
\hline ICU admission within $30 \mathrm{~d}$ of death & $10(11.9)$ \\
\hline Receipt of a palliative care consultation & $40(47.6)$ \\
\hline Receipt of palliative care $>30 \mathrm{~d}$ before death ${ }^{\mathrm{b}}$ & $18(22.0)$ \\
\hline Receipt of hospice services ${ }^{c}$ & $25(30.9)$ \\
\hline Hospice length of stay $>7 d^{a}$ & $18(22.5)$ \\
\hline \multicolumn{2}{|l|}{ Place of death } \\
\hline Home & $17(20.2)$ \\
\hline Hospital, rehabilitation facility, or nursing home & $31(36.9)$ \\
\hline Inpatient hospice & $6(7.1)$ \\
\hline Unknown & $30(35.7)$ \\
\hline \multicolumn{2}{|l|}{ Cause of death } \\
\hline Cancer progression & $58(69.1)$ \\
\hline CAR T-cell therapy complication & $4(4.8)$ \\
\hline Other causes & $6(7.1)$ \\
\hline Unknown & $16(19.1)$ \\
\hline
\end{tabular}

a4 patients with missing data.

b2 patients with missing data.

c3 patients with missing data.

hospitalized, $10.5 \%(n=2)$ received systemic therapy, and $21.1 \%(n=4)$ were admitted to the ICU. Among evaluable patients in this group, $12.5 \%(2 / 16)$ received hospice services, $21.1 \%(4 / 19)$ had a palliative care consultation, and $25 \%(3 / 12)$ died at home.

In multivariable analysis, receipt of bridging therapy (odds ratio [OR], 3.15; 95\% CI, 1.05-9.52; $P=.041$ ), index hospitalization LoS $>14$ days (OR, 4.76; 95\% CI, 1.47-15.37; $P=.009$ ), hospital admission within 3 months of CAR T-cell therapy (OR, 4.29; 95\% CI, 1.36-13.49; $P=.013$ ), and a diagnosis of indolent lymphoma transformed to DLBCL (OR, 9.83; 95\% CI, 1.66-58.24; $P=.012$ ) were all associated with a higher likelihood of hospitalization within 30 days of death (Table 4).

Additionally, we identified factors associated with likelihood of hospice referral utilizing multivariable logistic regression (Table 5). Controlling for age, sex, ECOG performance status, response, and lymphoma subtype, we found that palliative care consultation was associated with a greater likelihood of hospice referral (OR, 3.18; 95\% CI, 1.03-9.78; $P=.044$ ).

\section{Discussion}

In this study, we demonstrate that patients receiving CAR T-cell therapy experience substantial healthcare utilization, especially at EoL. More than one-quarter of CAR $\mathrm{T}$-cell recipients had one hospital readmission within 


\begin{tabular}{|lccc|}
\hline $\begin{array}{l}\text { Table 4. Factors Associated With Hospitalization } \\
\text { Within } 30 \text { Days of Death }\end{array}$ & & \\
\hline Variable & OR (95\% CI) & SE & P Value \\
\hline Age & $0.98(0.94-1.03)$ & 0.02 & .446 \\
\hline Female sex & $0.61(0.19-1.95)$ & 0.36 & .406 \\
\hline Received bridging therapy & $3.15(1.05-9.52)$ & 1.78 & $\mathbf{. 0 4 1}$ \\
\hline Complete response & $0.21(0.06-0.66)$ & 0.12 & $\mathbf{. 0 0 8}$ \\
\hline Length of stay >14 d & $4.76(1.47-15.37)$ & 2.85 & $\mathbf{. 0 0 9}$ \\
\hline $\begin{array}{l}\text { Hospital admission within 3 mo } \\
\text { of CAR T-cell infusion }\end{array}$ & $4.29(1.36-13.49)$ & 2.51 & $\mathbf{. 0 1 3}$ \\
\hline $\begin{array}{l}\text { Indolent lymphoma transformed } \\
\text { to DLBCL }\end{array}$ & $9.83(1.66-58.24)$ & 8.92 & $\mathbf{. 0 1 2}$ \\
\hline
\end{tabular}

Bold indicates statistically significant $P$ value.

Abbreviations: DLBCL, diffuse large B-cell lymphoma; OR, odds ratio

3 months of CAR T-cell therapy and $15.5 \%$ required ICU level of care. Notably, among deceased patients, $58.3 \%$ were hospitalized within 30 days of death, and most died in the hospital or healthcare facility. Unfortunately, most patients did not receive palliative care or hospice services. These findings underscore the intensity of EoL care for patients receiving CAR T-cell therapy and the need to optimize EoL care delivery in this population.

Healthcare utilization during the index hospitalization for CAR T-cell infusion was significant, with a median LoS exceeding 2 weeks and an ICU admission rate of nearly $13 \%$. Approximately one-quarter of patients experienced a rehospitalization within 3 months of CAR T-cell infusion; however, multiple rehospitalizations were uncommon, occurring in $<10 \%$ of patients. Moreover, most first rehospitalizations occurred within 30 days of the index CAR T-cell hospitalization and were most commonly due to uncontrolled symptoms. In our cohort, $5.9 \%$ of patients experienced grade $\geq 3$ CRS and $22.0 \%$ experienced grade $\geq 3$ ICANS, which was lower than the toxicity rates demonstrated with axicabtagene ciloleucel in the non-trial setting. ${ }^{20}$ This could be secondary to differences in the patient populations or due to the use of other CAR T-cell products with lower toxicity rates by some patients in our cohort. Our cohort had similar rates of response and 1-year survival to those reported in the published literature..$^{4,5,20-24}$ These findings suggest that early rehospitalization after CAR T-cell therapy is an important contributor to healthcare utilization in this population, and suggest that care transitions and symptom monitoring interventions at the time of discharge may be useful targets for future research.

We also demonstrate that patients receiving CAR T-cell therapy experience substantial healthcare utilization at EoL. Despite established evidence that most patients

\begin{tabular}{|lccc|}
\hline \multicolumn{4}{l}{ Table 5. Factors Associated With Hospice Referral } \\
Variable & OR (95\% CI) & SE & P Value \\
\hline Age & $1.02(0.98-1.07)$ & 0.02 & .349 \\
\hline Female sex & $0.36(0.10-1.30)$ & 0.24 & .119 \\
\hline Response & $0.25(0.08-0.78)$ & 0.14 & $\mathbf{. 0 1 7}$ \\
\hline Palliative care consultation & $3.18(1.03-9.78)$ & 1.82 & $\mathbf{. 0 4 4}$ \\
\hline ECOG performance status & $1.40(0.78-2.50)$ & 0.41 & .261 \\
\hline $\begin{array}{l}\text { HGBCL with MYC and BCL2 } \\
\text { and/or BCL6 translocations }\end{array}$ & $1.80(0.50-6.51)$ & 1.18 & .372 \\
\hline
\end{tabular}

Bold indicates statistically significant $P$ value.

Abbreviations: HGBLC, high-grade B-cell lymphoma; OR, odds ratio.

with cancer prefer to die at home and minimize time in the hospital at EoL, ${ }^{25,26}$ most patients receiving CAR T-cell therapy were hospitalized within the last 30 days of life, and most died in a hospital or healthcare facility. Palliative care and hospice services were infrequently used in this population, despite the demonstrated benefits of these services for improving quality of life and care for patients with cancer. ${ }^{27-34}$ The immense prognostic uncertainty along with the absence of a clear transition between the curative and palliative phase treatment in many patients receiving CAR T-cell therapy likely impacts EoL decision-making and contributes to high healthcare utilization at the EoL in this population. ${ }^{35}$ Prior studies have also shown high healthcare utilization at EoL and low rates of hospice and palliative care referrals in patients with hematologic malignancies. ${ }^{17,36,37}$ Interestingly, in our cohort, palliative care utilization was the only factor associated with hospice utilization, which may serve to optimize EoL care in this population. Given the demonstrated benefits of early palliative care integration for improving the quality of life and care for patients with solid tumors and hematologic malignancies, early referral to palliative care while pursuing curative-intent CAR T-cell therapy is a potentially helpful strategy to address the EoL care needs of this population.

We also determined that there are several clinical factors associated with the risk of hospitalization in the last 30 days of life. Factors such as the need for bridging therapy, a hospital readmission within 3 months of CAR T-cell infusion, and an index hospitalization $>14$ days are important clinical markers that identify a subset of patients at high-risk for acute clinical decompensation and worse EoL outcomes. These clinical factors can be used as triggers for additional interventions to optimize EoL care in this population. Importantly, these findings can help better inform both patients' and clinicians' expectations regarding the possible illness trajectory. 
Our study has several limitations worth considering. First, this study is a retrospective study of patients at 2 large academic sites, which lacked racial diversity, and thus our findings may not generalize to other populations. Second, we were limited to information about patients' healthcare utilization and EoL outcomes that were available in the EHR, and therefore our data may not have fully captured all healthcare utilization and EoL outcomes. Finally, our sample size limited the number of covariates we could analyze in multivariable logistic regression for EoL outcomes; thus, our model may not fully account for all possible confounders. Future research studies should assess healthcare utilization and EoL outcomes in a diverse population of patients undergoing CAR T-cell therapy to ensure the generalizability of these findings. Future efforts should also explore costs of care at EoL in this population.

\section{Conclusions}

We demonstrated that approximately one-quarter of patients receiving CAR T-cell therapy experience a hospitalization within 3 months of their CAR T-cell infusion, and most experience high healthcare utilization at EoL. We also identified salient factors associated with the risk for hospitalization within 30 days of death. Our findings underscore the need to develop transitional care and EoL care interventions to improve the quality of care for patients receiving CAR T-cell therapy.

Submitted September 8, 2020; final revision received October 9, 2020; accepted for publication October 26, 2020. Published online March 11, 2021.

Author contributions: Study concept and design: All authors. Data acquisition: All authors. Data analysis and interpretation: All authors. Manuscript preparation: All authors. Critical revision: All authors. Final approval of manuscript: All authors.

Disclosures: Dr. Jacobson has reported receiving grant/research support from Kite Pharma/Gilead, and serving on an advisory board for Kite Pharma/ Gilead, Novartis, Bristol-Myers Squibb/Celgene, Nkarta, Precision Biosciences, and Lonza. Dr. Frigault has reported serving as a consultant for and receiving grant/research support from Novartis and Kite Pharma. The remaining authors have reported that they have not received any financial consideration from any person or organization to support the preparation, analysis, results, or discussion of this article.

Funding: This work was supported by funding from the Leukemia and Lymphoma Society.

Correspondence: P. Connor Johnson, MD, Department of Medicine, Division of Hematology \& Oncology, Massachusetts General Hospital Cancer Center, 55 Fruit Street, Yawkey 7B, Boston, MA 02114.

Email: pcjohnson@mgh.harvard.edu

\section{References}

1. Naldini L. Gene therapy returns to centre stage. Nature $2015 ; 526$ 351-360.

2. Maus MV, Fraietta JA, Levine BL, et al. Adoptive immunotherapy for cancer or viruses. Annu Rev Immunol 2014;32:189-225.

3. Maude SL, Frey N, Shaw PA, et al. Chimeric antigen receptor T cells for sustained remissions in leukemia. N Engl J Med 2014;371:1507-1517.

4. Neelapu SS, Locke FL, Bartlett NL, et al. Axicabtagene ciloleucel CAR T-cell therapy in refractory large B-cell lymphoma. N Engl J Med 2017; 377:2531-2544.

5. Schuster SJ, Bishop MR, Tam CS, et al. Tisagenlecleucel in adult relapsed or refractory diffuse large B-cell lymphoma. N Engl J Med 2019;380: 45-56.

6. Lee DW, Santomasso BD, Locke FL, et al. ASTCT consensus grading for cytokine release syndrome and neurologic toxicity associated with immune effector cells. Biol Blood Marrow Transplant 2019;25: 625-638

7. Neelapu SS, Tummala S, Kebriaei P, et al. Chimeric antigen receptor T-cell therapy_assessment and management of toxicities. Nat Rev Clin Onco 2018;15:47-62

8. Neelapu SS, Tummala S, Kebriaei P, et al. Toxicity management after chimeric antigen receptor T cell therapy: one size does not fit 'ALL.' Nat Rev Clin Oncol 2018;15:218.

9. Liu D, Zhao J. Cytokine release syndrome: grading, modeling, and new therapy. J Hematol Oncol 2018;11:121.

10. Acharya UH, Dhawale T, Yun S, et al. Management of cytokine release syndrome and neurotoxicity in chimeric antigen receptor (CAR) T cell therapy. Expert Rev Hematol 2019;12:195-205.

11. Crump M, Neelapu SS, Faroog $U$, et al. Outcomes in refractory diffuse large B-cell lymphoma: results from the international SCHOLAR-1 study.Blood 2017;130:1800-1808.

12. El-Jawahri A, Keenan T, Abel GA, et al. Potentially avoidable hospital admissions in older patients with acute myeloid leukaemia in the USA: a retrospective analysis. Lancet Haematol 2016;3:e276-283.

13. Brooks GA, Jacobson JO, Schrag D. Clinician perspectives on potentially avoidable hospitalizations in patients with cancer. JAMA Oncol 2015;1: 109-110.

14. Hui D, Kim SH, Roquemore J, et al. Impact of timing and setting of palliative care referral on quality of end-of-life care in cancer patients. Cancer 2014;120:1743-1749.
15. Nipp RD, El-Jawahri A, D'Arpino SM, et al. Symptoms of posttraumatic stress disorder among hospitalized patients with cancer. Cancer 2018; 124:3445-3453.

16. Bursac Z, Gauss $C H$, Williams DK, et al. Purposeful selection of variables in logistic regression. Source Code Biol Med 2008;3:17.

17. Odejide OO, Cronin AM, Earle CC, et al. Hospice use among patients with lymphoma: impact of disease aggressiveness and curability. J Natl Cancer Inst 2015:108:108.

18. Pinnix CC, Gunther JR, Dabaja BS, et al. Bridging therapy prior to axicabtagene ciloleucel for relapsed/refractory large B-cell lymphoma. Blood Adv 2020;4:2871-2883.

19. Mercadante S, Masedu F, Valenti M, et al. The characteristics of advanced cancer patients followed at home, but admitted to the hospital for the last days of life. Intern Emerg Med 2016;11:713-718.

20. Jacobson CA, Hunter BD, Redd R, et al. Axicabtagene ciloleucel in the non-trial setting: outcomes and correlates of response, resistance, and toxicity. J Clin Oncol 2020;38:3095-3106.

21. Abramson JS, Palomba ML, Gordon LI, et al. Pivotal safety and efficacy results from Transcend NHL 001, a multicenter phase 1 study of lisocabtagene maraleucel (liso-cel) in relapsed/refractory (R/R) large B cell lymphomas. Blood 2019;134(Suppl 1):241.

22. Jaglowski $\mathrm{S}, \mathrm{Hu} \mathrm{ZH}$, Zhang $\mathrm{Y}$, et al. Tisagenlecleucel chimeric antigen receptor (CAR) T-cell therapy for adults with diffuse large B-cell lymphoma (DLBCL): real world experience from the Center for International Blood \& Marrow Transplant Research (CIBMTR) Cellular Therapy (CT) Registry [abstract]. Blood 2019;134(Suppl 1):766. Abstract 627.

23. Locke FL, Ghobadi A, Jacobson CA, et al. Long-term safety and activity of axicabtagene ciloleucel in refractory large B-cell lymphoma (ZUMA-1): a single-arm, multicentre, phase 1-2 trial. Lancet Oncol 2019;20:31-42.

24. Nastoupil LJ, Jain MD, Feng L, et al. Standard-of-care axicabtagene ciloleucel for relapsed or refractory large B-cell lymphoma: results from the US lymphoma CAR T Consortium. J Clin Oncol 2020;38: 3119-3128.

25. Beccaro M, Costantini M, Giorgi Rossi P, et al. Actual and preferred place of death of cancer patients. Results from the Italian survey of the dying of cancer (ISDOC). J Epidemiol Community Health 2006;60:412-416.

26. Higginson IJ, Sen-Gupta GJ. Place of care in advanced cancer: a qualitative systematic literature review of patient preferences. J Palliat Med 2000;3:287-300 
27. Bakitas M, Lyons KD, Hegel MT, et al. Effects of a palliative care intervention on clinical outcomes in patients with advanced cancer: the Project ENABLE II randomized controlled trial. JAMA 2009;302:741-749.

28. Wright AA, Keating NL, Balboni TA, et al. Place of death: correlations with quality of life of patients with cancer and predictors of bereaved caregivers' mental health. J Clin Oncol 2010;28:4457-4464.

29. Wright AA, Zhang B, Ray A, et al. Associations between end-of-life discussions, patient mental health, medical care near death, and caregiver bereavement adjustment. JAMA 2008;300:1665-1673.

30. El-Jawahri A, LeBlanc $T$, VanDusen $\mathrm{H}$, et al. Effect of inpatient palliative care on quality of life 2 weeks after hematopoietic stem cell transplantation: a randomized clinical trial. JAMA 2016;316:2094-2103.

31. El-Jawahri A, Traeger L, Greer JA, et al. Effect of inpatient palliative care during hematopoietic stem-cell transplant on psychological distress 6 months after transplant: results of a randomized clinical trial. J Clin Oncol 2017;35:3714-3721.
32. Temel JS, Greer JA, Muzikansky A, et al. Early palliative care for patients with metastatic non-small-cell lung cancer. N Engl J Med 2010;363: 733-742.

33. Bakitas MA, Tosteson TD, Li Z, et al. Early versus delayed initiation of concurrent palliative oncology care: patient outcomes in the ENABLE III randomized controlled trial. J Clin Oncol 2015;33:1438-1445.

34. Zimmermann C, Swami N, Krzyzanowska M, et al. Early palliative care for patients with advanced cancer: a cluster-randomised controlled trial. Lancet 2014;383:1721-1730.

35. El-Jawahri A, Nelson AM, Gray TF, et al. Palliative and end-of-life care for patients with hematologic malignancies. J Clin Oncol 2020;38:944-953.

36. Sexauer A, Cheng MJ, Knight $L$, et al. Patterns of hospice use in patients dying from hematologic malignancies. J Palliat Med 2014;17:195-199.

37. Hui D, Didwaniya N, Vidal M, et al. Quality of end-of-life care in patients with hematologic malignancies: a retrospective cohort study. Cancer 2014;120:1572-1578.

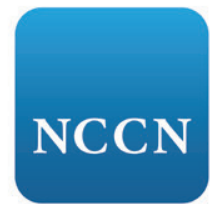

March 31 - April 2
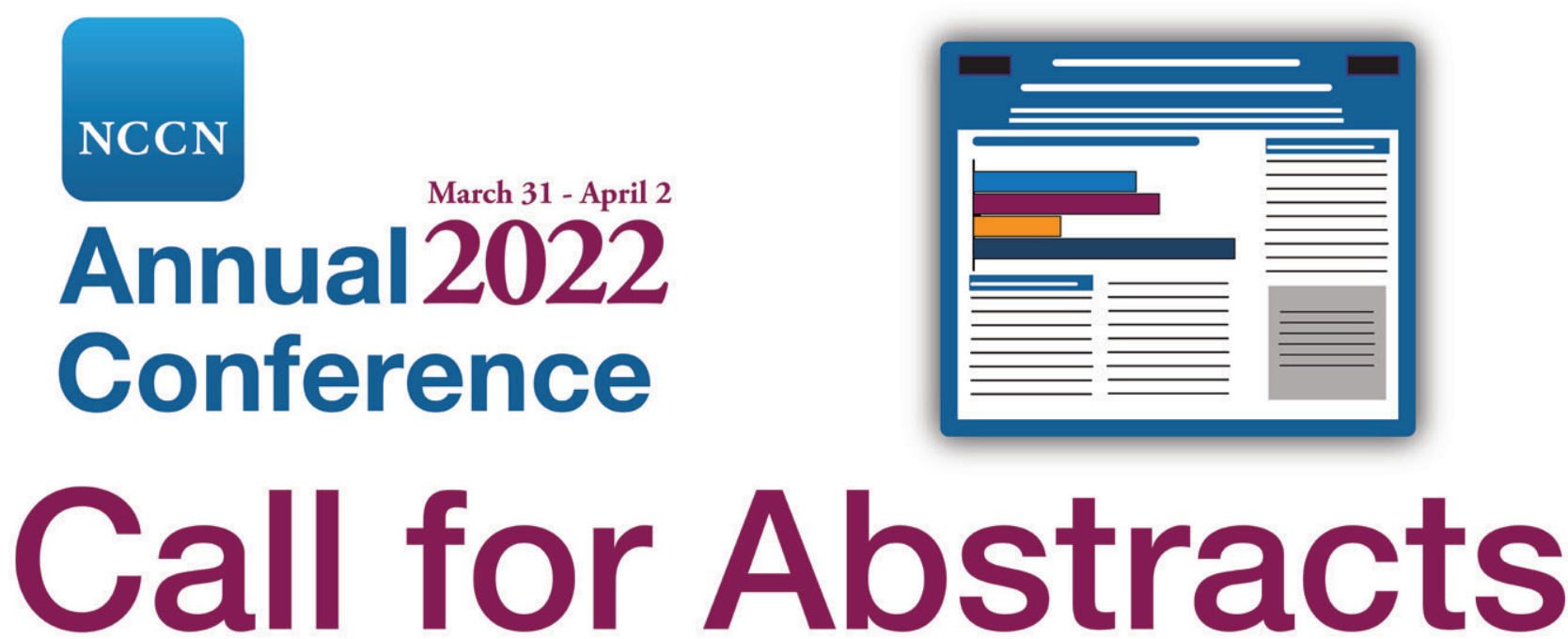

Submit now: NCCN.org/conference | Deadline: 11.10.21

JNCCN-N-0300-0821 